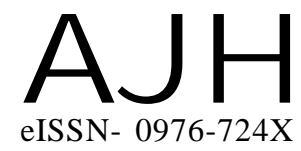

Article history :

Received : 23.10.2015

Revised : 11.11 .2015

Accepted : 23.11.2015
Author for correspondence

College of Horticulture and Forestry (Dr. Y.S. Parmar University of Horticulture and Forestry), Khagal, HAMIRPUR (H.P.) INDIA

\title{
Effect of surface wetness and duration of low temperature exposure on frost damage in sub- tropical fruit species
}

\section{SHASHI K. SHARMA}

ABSTRACT : Frost is the most devastating weather hazard for horticultural growth in the NW India. For mitigation of its impact the information on the process of freeze initiation a spread and the factors involved in aggravating the damage is of utmost importance. Studies were conducted at COHF Neri under controlled and radiation frost customized environment. REL was the measure adopted for accessing the damage. Two leaf surface wetness levels (wet and dry) along with three durations of low temperature $\left(-2^{\circ} \mathrm{C}\right)$ exposure 2 hours, 4 hours and 6 hours were taken under a Completely Randomized Factorial experiment conducted on container grown ten subtropical fruit species. Leaf surface wetness was more critical in case of mango and litchi whereas in case guava, jamun, karonda, galgal, lime extrinsic ice nucleation was not very important in inducing internal damage to the leaf tissues. Duration of low temperature exposure aggravated the damage level in almost all the species. In case of papaya and jackfruit higher susceptibility of these species to frost induced freeze damage the impact of frost duration was not found to be significant. The relative order off susceptibility of different species was observed to be as: Loquat, galgal, lime, guava, karonda, jamun, litchi, mango, jackfruit, papaya in the increasing order.

KEY WORDS : Frost damage, Mango, Litchi, Freezing susceptibility, Relative electrolyte leakage

HOW TO CITE THIS ARTICLE : Sharma, Shashi K. (2015). Effect of surface wetness and duration of low temperature exposure on frost damage in sub-tropical fruit species. Asian J. Hort., 10(2) : 272-277. 